\title{
Der Kliniker ist gefragt
}

\section{Heinz-Michael Just}

Surveillance will gekonnt sein...

Surveillance muss gewollt sein...

nur gemusst - sollte sie nicht sein!

Mit Inkrafttreten des Infektionsschutzgesetzes (IfSG) ist eine Surveillance zur Verpflichtung geworden - mit allem Für und Wider. Aber eben nur „eine“ und nicht „die“! Die zu beobachtenden Varianten in der Umsetzung zeigen deutlich Nutzen wie Gefahren auf. Nicht überall wurde verstanden, was die Initiatoren wollten: allen, die an Patienten invasiv und damit risikobeladen tätig sind, ein Instrument an die Hand geben, um ihr eigenes Tun verantwortungsvoll und selbstkritisch zu begleiten. Nicht selten wird dies jedoch gar nicht gewollt oder die Surveillance wird lediglich als eine zu erfüllende, vielleicht sogar lästige Pflicht angesehen, der man sich am einfachsten dadurch entledigt, dass man streng 1 zu 1 - und damit automatisch unkritisch - entsprechende Empfehlungen in die Praxis umzusetzen versucht, eventuell gar nur mit dem Ziel, das eigene Gesundheitsamt zufriedenzustellen. Die Hilfe zur Selbsthilfe mit dem Kürzel „KISS“ wird hierbei dann als Feigenblatt missbraucht - in manchen Bundesländern sogar verpflichtend auf der Basis einer Verordnung.

Diese KISS-Methode wurde in Deutschland durch das Nationale Referenzzentrum für Surveillance zur Erfassung mit dem Ziel etabliert, eine einheitliche, nach standardisierten Kriterien durchzuführende Erfassung zu ermöglichen. Als Ergebnis eines solchermaßen einheitlichen Vorgehens auf freiwilliger Basis erhofft man sich den Aufbau eines Datenpools, der primär zur (fortlaufenden) Beurteilung der eigenen Daten, in zweiter Linie aber auch für ein „Benchmarking“ d. h. Vergleich mit anderen, vergleichbaren Kliniken/ Abteilungen - geeignet sein soll. Voraussetzung für Letzteres sind einheitliche Erfassungskriterien und ehrliche Daten, für deren Qualität die Erfasser selbst verantwortlich sind. Nur dies ermöglicht die für ein solches Benchmarking zwingend notwendige Qualität des Datenpools.
Unter den für die Erfassung verwendeten Infektionsdefinitionen ist der Erregernachweis ein wesentlicher Bestandteil, womit die mikrobiologische Diagnostik als Grundlage für ein effektives Infektionsmanagement weiter an Bedeutung gewonnen hat.

In dem Beitrag von R. Ziegler in diesem Heft wird an Beispielen dargelegt, wie irreführend mikrobiologische Befunde bei unkritischer Interpretation sein können und welch weitreichende Konsequenzen dies haben kann. Eine Optimierung erscheint dringend erforderlich, aber nur realisierbar, wenn die Zusammenarbeit zwischen behandelndem Arzt und klinischem Mikrobiologen in der im Beitrag beschriebenen Weise intensiviert werden kann. Als ganz wesentlich hierfür zeigt sich zukünftig die klinische Zuordnung der isolierten Keime zu „Kontaminanten“, „Kolonisationskeimen“ und „Infektionserregern“. Nur Letztere sollten in einer Keim- und Resistenzstatistik Verwendung finden, die üblicherweise dem Kliniker als Basis für eine sogenannte „kalkulierte“ Primärtherapie bei vergleichbaren Indikationen dient. Diese Zuordnung kann aber nur vom Kliniker selbst geleistet werden. Derart bereinigte Analysen dürften sich jedoch im Ergebnis grundlegend von den bislang übermittelten Statistiken unterscheiden, insbesondere, wenn sie nach Infektionsarten getrennt ausgewertet werden. Diese Analysen wären damit sicher besser als Grundlage für eine kalkulierte Antibiotikatherapie vor Kenntnis des Infektionserregers geeignet. Würden sich dann vielleicht auch die gegenwärtigen Therapieversager trotz „adäquater Antibiose“ erklären? Die Kostenrelevanz läge auf der Hand, was die Kooperationsbereitschaft des Klinikers zusätzlich fördern dürfte.

Der $\S 23$ verpflichtet darüber hinaus aber auch zur systematischen Erfassung und Bewertung von Infektionserregern mit speziellen Antibiotikaresistenzen. Das Ziel hierbei ist es, den behandelnden Arzt zum kritischen Hinterfragen seiner Antibiotikaverordnungsgewohnheiten anzuregen. Das Ergebnis kann ebenfalls eine systematische „Surveillance“ stationsbezogener Antibiotikaverordnungen sein; mit einer 
vergleichenden Verlaufsanalyse von Antibiotikaverbrauch und Resistenzentwicklung - der Folge ungerechtfertigter Antibiotikagaben - in definierten Bereichen eines Krankenhauses (SARI).

E. Meyer verweist in ihrem Beitrag auf das Problem von „Outcome-Parametern“ einer Antibiotika-Surveillance. Auch sie macht deutlich, wie schwierig ein Vergleich der eigenen Daten mit den Vergleichsdaten anderer Intensivstationen ist, und dass hier noch dringlicher die fachärztliche, kritische Interpretation der eigenen Daten vor einer Bewertung vonnöten ist. Insbesondere die sich zunehmend verändernden Eigenschaften von Infektionserregern (s. auch den Beitrag von $\mathrm{H}$. Linde und $\mathrm{N}$. Lehn in diesem Heft) und damit einhergehend die schwieriger oder teurer werdenden Therapien belegen, dass Krankenhaushygiene heute eine andere Herangehensweise erfordert, als über Konzepte von rein und unrein, albernen roten Strichen auf Fußböden (von manchen Aufsichtsbehörden immer noch gefordert), flächendeckender Desinfektionsmittelanwendung, Schleusen oder Klimaanlagen.

Durch die Beiträge in diesem Heft wird erneut deutlich, warum Krankenhaushygieniker infektiologisch ausgebildete Ärzte (Humanmediziner) sein müssen, die kontinuierlich in Kontakt mit ihren klinischen Kollegen stehen und regelmäßig bei Visiten mitgehen. Reine Surveillance-Statistik im Kämmerlein ist ebenso wenig hilfreich wie rein theoretische „Hygieneemp- fehlungen“ aus klinikfernen Institutionen. Gefragt sind Handlungs- und Verhaltensregeln, die sich ausschließlich an den Erregereigenschaften und der persönlichen Situation des Patienten zu orientieren haben: KISS und SARI als Fundamente für ein Infektionsaudit durch qualifizierte Krankenhaushygieniker mit dem Kliniker als zentral Verantwortlichen.

Solche Hygieneempfehlungen bedürfen in Zeiten knapper Kassen aber einer situationsgerechten Anwendung, was häufig Modifikationen erforderlich macht. In dem Beitrag von I. Kappstein werden nicht nur für einen Bereich die hierfür maßgeblichen Aspekte aufgezeigt und diskutiert, sondern auch darauf hingewiesen, dass nicht nur die Anwender, sondern auch die Autoren von Empfehlungen und vergleichbaren Regelwerken mit jener Sorgfalt zu Werke gehen müssen, die es den Adressaten ermöglicht, den Aussagen das erforderliche Vertrauen entgegenzubringen. Bei der Erstellung gehören hierzu nicht nur eine umfassende und kritische Literaturrecherche als Grundlage evidenzbasierter Empfehlungen, sondern auch klare Evidenzkategorien mit nachvollziehbaren wissenschaftlich belegbaren Definitionen (reine Konsensusempfehlungen sollten nur noch ausnahmsweise formuliert werden). Die am Ende des Beitrags aufgeführten Beispiele verdeutlichen, welche Fallstricke zu beachten sind, und warum Empfehlungen immer nur Hilfestellung sein können und nie Ersatz für eigenverantwortliches situationsangepasstes Handeln. 